Article

\title{
An Azimuth Signal-Reconstruction Method Based on Two-Step Projection Technology for Spaceborne Azimuth Multi-Channel High-Resolution and Wide-Swath SAR
}

\author{
Ning Li ${ }^{1,2,3} \mathbb{D}$, Hanqing Zhang ${ }^{1,2,3}$, Jianhui Zhao ${ }^{1,2,3, *}$, Lin $\mathrm{Wu}^{1,2,3} \mathbb{D}$ and Zhengwei Guo ${ }^{1,2,3}$ \\ 1 College of Computer and Information Engineering, Henan University, Kaifeng 475004, China; \\ hedalining@henu.edu.cn (N.L.); zhanghanqing@henu.edu.cn (H.Z.); henuwl@henu.edu.cn (L.W.); \\ gzw@henu.edu.cn (Z.G.) \\ 2 Henan Engineering Research Center of Intelligent Technology and Application, Henan University, \\ Kaifeng 475004, China \\ 3 Henan Key Laboratory of Big Data Analysis and Processing, Henan University, Kaifeng 475004, China \\ * Correspondence: jhzhao@henu.edu.cn
}

\section{check for}

updates

Citation: Li, N.; Zhang, H.; Zhao, J.; $\mathrm{Wu}, \mathrm{L}$; Guo, Z. An Azimuth

Signal-Reconstruction Method Based on Two-Step Projection Technology for Spaceborne Azimuth

Multi-Channel High-Resolution and Wide-Swath SAR. Remote Sens. 2021, 13, 4988. https://doi.org/10.3390/ rs13244988

Academic Editors: Kun-Shan Chen, Saibun Tjuatja and Xinwu Li

Received: 14 November 2021

Accepted: 6 December 2021

Published: 8 December 2021

Publisher's Note: MDPI stays neutral with regard to jurisdictional claims in published maps and institutional affiliations.

Copyright: (c) 2021 by the authors. Licensee MDPI, Basel, Switzerland. This article is an open access article distributed under the terms and conditions of the Creative Commons Attribution (CC BY) license (https:/ / creativecommons.org/licenses/by/ $4.0 /)$.

\begin{abstract}
Azimuth non-uniform signal-reconstruction is a critical step for azimuth multi-channel high-resolution wide-swath (HRWS) synthetic aperture radar (SAR) data processing. However, the received non-uniform signal has noise in the actual azimuth multi-channel SAR (MCSAR) operation, which leads to the serious reduction in the signal-to-noise ratio (SNR) of the results processed by a traditional reconstruction algorithm. Aiming to address the problem of reducing the SNR of the traditional reconstruction algorithm in the reconstruction of non-uniform signal with noise, a novel signal-reconstruction algorithm based on two-step projection technology (TSPT) for the MCSAR system is proposed in this paper. The key part of the TSPT algorithm consists of a two-step projection. The first projection is to project the given signal into the selected intermediate subspace, spanned by the integer conversion of the compact support kernel function. This process generates a set of sparse equations, which can be solved efficiently by using the sparse equation solver. The second key projection is to project the first projection result into the subspace of the known sampled signal. The secondary projection can be achieved with a digital linear translation invariant (LSI) filter and generate a uniformly spaced signal. As a result, compared with the traditional azimuth MCSAR signal-reconstruction algorithm, the proposed algorithm can improve SNR and reduce the azimuth ambiguity-signal-ratio (AASR). The processing results of simulated data and real raw data verify the effectiveness of the proposed algorithm.
\end{abstract}

Keywords: signal-reconstruction; high-resolution wide-swath (HRWS); synthetic aperture radar (SAR); non-uniform sampling; two-step projection technology (TSPT); signal-to-noise ratio (SNR); azimuth ambiguity-signal-ratio (AASR)

\section{Introduction}

As a mature remote-sensing technology, synthetic aperture radar (SAR) can realize all-day and all-weather imaging of the Earth's surface, and it plays a vital role in Earth observation and remote sensing [1-5]. With the development of remote-sensing application demand in recent years, SAR is expected to observe a wide range of scenes while having the ability to detect small targets. However, due to the limitation of the minimum antenna area, the conventional single-channel SAR systems cannot meet the requirements of simultaneous high resolution and wide swath [6,7]. To obtain high azimuth resolution, a small azimuth antenna length is needed to obtain a wide Doppler bandwidth, and a high pulse repetition frequency $(P R F)$ is needed to avoid azimuth spectrum ambiguity [8-11]. However, a wide-range swath requires low PRF to avoid range ambiguity. Therefore, high azimuth resolution and wide swath are irreconcilable contradictions in the conventional single-channel SAR system. 
To solve the above contradiction, the concept of the azimuth multi-channel is proposed as a potential solution [12-15]. The multi-channel SAR (MCSAR) has more spatial degrees of freedom than the conventional single-channel SAR by setting a series of receiving apertures in azimuth. An azimuth MCSAR system transmits a signal with low PRF to achieve a wide swath, and at the same time, all the apertures receive signals to increase the sampling rate equivalently, alleviating the constraint of the minimum antenna area of the conventional single-aperture spaceborne SAR [8,16-18]. When the actual PRF of the MCSAR system satisfies the uniform sampling requirement, i.e.,

$$
P R F_{u n i}=\frac{2 v_{s}}{N d_{a}}
$$

where $v_{S}$ denotes the velocity of the SAR carrier, $N$ represents the number of the channels, and $d_{a}$ is the displacement between the adjacent receivers, then the uniformly sampled azimuth signal can be generated by simply rearranging the MCSAR echo. However, PRF is difficult to satisfy the uniform sampling condition in azimuth MCSAR system design. Non-uniform sampling will cause azimuth Doppler ambiguity in the echo signal received by each channel. The comparison between uniform sampling and non-uniform sampling has been shown in Figure 1, where a triangle, a circle and a square represent the echo signal received by the first channel, the second channel and the third channel, respectively. If azimuth multi-channel SAR echo data are directly imaged without reconstruction, the quality of the image will be greatly reduced. Therefore, to achieve unambiguous images, Doppler spectrum reconstruction is necessary.

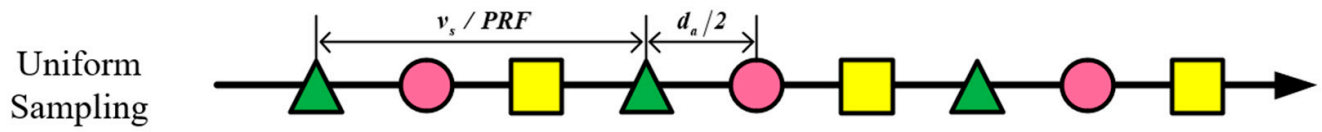

(a)

Nonuniform
Sampling

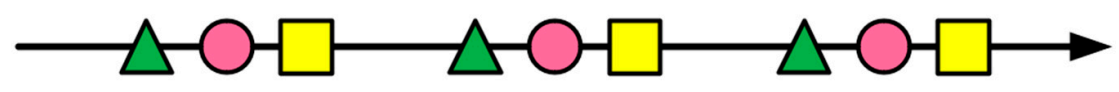

(b)

Figure 1. (a) Uniform sampling; (b) Non-uniform sampling.

At present, several effective algorithms [19-25] have been proposed to solve the problem of non-uniform sampling reconstruction for azimuth multi-channel spaceborne SAR. The representative azimuth MCSAR signal-reconstruction algorithms are the digital beamforming (DBF) algorithm proposed by Kriger et al. $[3,9,25]$ and the space-time adaptive processing (STAP) algorithm proposed by Li et al. $[19,20]$. The DBF algorithm solves a set of linear equations to explicitly reconstruct the Doppler spectrum from the aliased multi-channel signals. In contrast, the STAP algorithm used a minimum variance distortionless response (MVDR) beamformer to suppress the azimuth ambiguity. If azimuth MCSAR echo data are an idea band-limited signal without noise, the performance of the two algorithms is consistent, as demonstrated in [25]. However, in the above two signal-reconstruction algorithms, the ambiguity number and ambiguity index are considered to be fixed without changing the baseband Doppler frequency, which will lead to a reduction in the SNR of the reconstructed signal in the case of non-uniform sampling. In fact, the ambiguity information will change with the Doppler frequency, which also complicates the construction of the steering vector. Aiming at solving the above problem, several methods are proposed to improve the DBF and STAP reconstruction algorithms to improve the performance of azimuth ambiguity suppression [21-24]. The improved DBF (IDBF) algorithm proposed in [21] defined the equivalent sampling interval to calculate the equivalent reconstructed Doppler bandwidth (ERDB). The IDBF algorithm overcomes the 
shortcomings of Kriger's DBF algorithm to some extent, but at the cost of increasing the azimuth ambiguity-signal-ratio (AASR) and decreasing the azimuth resolution. Inspired by the IDBF algorithm, Liu et al. proposed the ImpMMSE reconstruction algorithm in the literature [22]. The ImpMMSE algorithm calculates ERDB by weighted operations and uses the minimum mean square error (MMSE) criterion to suppress azimuth ambiguity, which improved AASR. In the literature [23], the baseband Doppler center and ERDB were calculated according to the Capon spectrum estimation method. In the above-mentioned improved DBF method, the calculation of ERDB and the operation of ambiguity index screening (AIS) depend on the system parameters. To solve the problem of the insufficient suppression of strong scattering points by the STAP algorithm, Zhang et al. proposed a Doppler ambiguity suppression algorithm based on multiple Doppler direction constraints to eliminate the ambiguity component in the Doppler domain [24].

This paper innovatively proposes the idea of sparse uniform resampling to solve the problem of azimuth MCSAR non-uniform signal-reconstruction [26]. A two-step projection technique (TSPT) is derived, and the general method of TSPT algorithm processing is summarized. The main idea of the TSPT algorithm is to construct an interim subspace that is designed to reconstruct an azimuth MCSAR signal. The signal-reconstruction process of the TSPT algorithm includes two important projection sequences. The first projection projects the given azimuth MCSAR non-uniform frequency domain signal into the intermediate subspace formed by the integer translation that tightly supports the kernel function. After the first step of projection, a sparse equation system can be derived, which describes the relationship between the non-uniform sample and the coefficient vector of the auxiliary subspace. The second projection uses a digital linear shift invariant (LSI) filter to project the result of the first projection into the subspace where the sampled signal is known. After completing the above operations, the evenly spaced signal can be generated in the frequency domain. Finally, the reconstructed image can be obtained by performing the Fourier transform on a uniform sample. The proposed method can effectively suppress the ambiguity of the azimuth Doppler spectrum caused by non-uniformity and maintain a good SNR.

The succeeding organization of this paper is as follows. Section 2 gives some characteristics of the signal model of azimuth MCSAR. Section 3 reviews the traditional reconstruction algorithm and its improved algorithm. Section 4 details the derivation of the proposed algorithm. Section 5 shows the experimental and analytical results. Finally, Section 6 provides conclusions.

\section{Signal Model}

The MCSAR system, with a planar antenna array, is composed of $N$ sub-apertures uniformly placed in azimuth. Figure 2 shows the geometry of a typical azimuth five-channel SAR system, where $W_{g}$ and $h_{z}$ denote the swath width and platform height, respectively. In the Cartesian geometric coordinate system in Figure 2, the $X$-axis is the radar flight direction, the $\mathrm{Y}$-axis points to the left and is perpendicular to the $\mathrm{X}$-axis, and the $\mathrm{Z}$-axis is far from the center of the Earth. The platform moves at speed $v_{s}$ along the $\mathrm{X}$-axis. The mid-aperture of the azimuth five-channel SAR system is the transmitting and receiving aperture. The remaining apertures are the receiving apertures, that is, one transmitting and five receiving in azimuth. The echo data of each channel are compensated by the constant phase term, which is equivalent to the data transmitted and received by the corresponding effective phase center (EPC) $[9,24]$. As shown in Figure 2, the EPC is located in the middle position of the transmitter and corresponding receiver. Therefore, the distance between adjacent EPCs is $d_{a}$. 


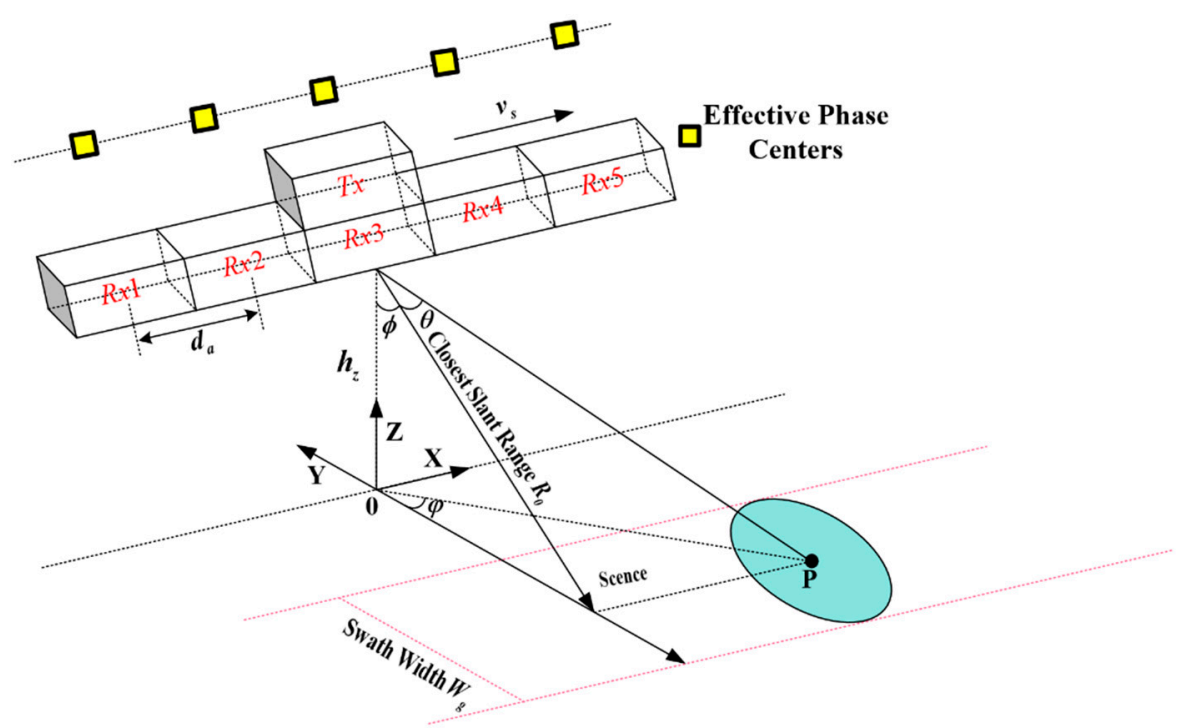

Figure 2. Geometry of an azimuth multi-channel SAR system.

Considering an azimuth MCSAR system composed of $N$ channels uniformly arranged in azimuth, among the $N$ channels, the middle channel is selected as the reference channel to transmit the signal, and all of the $N$ channels receive echo simultaneously. Assuming that the azimuth slow time $\eta=0$, the observed target $P$ is directly above the transmitter aperture, and $R_{0}$ represents the closest slant range from the target $P(x, y, z)$ in the scene to the movement trajectory of the platform. $R_{m}$ represents the instantaneous slant range from the transmitting antenna to the target $P$ and then to the receiving channel $m$. The relationship between the three angles can be expressed as

$$
\sin \theta=\sin \varphi \sin \phi
$$

where $\varphi$ indicates the azimuth angle, $\phi$ represents the depression angle, and $\theta$ means the cone angle. Assuming that the coordinate position of EPC $m$ at $\eta=0$ is $\left(\Delta x_{m}, 0, h_{z}\right)$, then the coordinate position of the $m$ th EPC is $\left(v_{s} \eta+\Delta x_{m}, 0, h_{z}\right) . d_{a}$ is the azimuth offset between EPC $m$ and the reference EPC, and $\Delta x_{m}=(m-(N+1) / 2) d_{a}$. For a single point target, after demodulation, the two-dimensional time-domain echo model of the $m$ th sub-aperture can be expressed as $[24,27,28]$

$$
\begin{aligned}
& x_{m}(\tau, \eta)=\iint \sigma(x, y) g\left(\tau-\frac{2 R_{m}(x, y, \eta)}{c}\right) \\
& a\left(\eta-\frac{x-\Delta x_{m}-x_{0}}{v_{s}}\right) \exp \left(-j \frac{4 \pi}{\lambda} R_{m}(x, y, \eta)\right) d x d y
\end{aligned}
$$

where

$$
R_{m}(x, y, \eta)=\sqrt{\left(v_{s} \eta-\Delta x_{m}-x\right)^{2}+R_{0}^{2}(x, y)}
$$

where $\tau$ represents range fast time, $g(\tau)$ is the pulse signal transmitted by the SAR system, $c$ is the velocity of light, $a(\eta)$ indicates the azimuth antenna pattern, $\sigma(x, y)$ represents the scattering characteristics of the target at $(x, y)$, and $R_{0}(x, y)=\sqrt{y^{2}+h_{z}^{2}}$ indicates the shortest slant range of the target $(x, y)$ from the radar platform. Carrying out the azimuth Fourier transform on (3), the echo signal model in the range-Doppler domain can be obtained as

$$
x_{m}\left(\tau, f_{a}\right)=s\left(\tau, f_{a}\right) \exp \left(j 2 \pi f_{a} \frac{\Delta x_{m}}{v_{s}}\right)
$$


where

$$
\begin{aligned}
s\left(\tau, f_{a}\right) & =\iint \sigma(x, y) G\left(\tau-2 R_{0}(x, y) / c \sqrt{1-\frac{c^{2} f_{a}^{2}}{4 v_{s}^{2} f_{0}^{2}}}\right) \\
& \times A\left(f_{a}-f_{d c}\right) \exp \left(-j \frac{4 \pi R_{0}(x, y) D\left(f_{a}, v_{s}\right) f_{0}}{c}\right) \\
& \times \exp \left(-j 2 \pi f_{a} \frac{\Delta x_{m}}{v_{s}}\right) d x d y
\end{aligned}
$$

$G(\tau)$ indicates the range complex signal and $A\left(f_{a}-f_{d c}\right)$ represents the Doppler spectrum envelope with $f_{d c}$ as the Doppler center.

When the PRF is higher than the Doppler bandwidth of each channel echo, each Doppler frequency unit contains numerous scene echo signals with the same cone angle $\theta$, that is, the Doppler frequency $f_{a}$ has a linear relationship with the cone angle $\theta[29,30]$, i.e.,

$$
f_{a}=\frac{2 v_{s} \sin \theta}{\lambda}
$$

To obtain HRWS and range unambiguity SAR images, the signals received by each channel are usually under-sampled in azimuth. The MCSAR signal causes Doppler aliasing due to azimuth under-sampling, so each Doppler unit contains echo signals of different azimuth frequencies, and the minimum interval between these frequencies is PRF. Figure 3 shows the unambiguous Doppler spectrum and the ambiguous Doppler spectrum, where the Doppler ambiguity number $I=3$. The dashed line in Figure 3 shows that there is no Doppler aliasing when the PRF is greater than the Doppler bandwidth of the echo signal. Each Doppler unit corresponds to the echo signal from the same cone angle $\theta$. The solid line represents Doppler aliasing, and each Doppler unit contains an echo signal from different cone angles. In the azimuth MCSAR system, due to the azimuth under-sampling, the echo signal of the $m$ th sub-aperture in the range-Doppler domain can be rewritten as

$$
x_{m}\left(\tau, f_{a}\right)=\sum_{i=i_{\min }}^{i_{\max }} \exp \left(j 2 \pi\left(f_{a}+i \cdot P R F\right) \frac{\Delta x_{m}}{v_{s}}\right) \cdot s\left(\tau, f_{a}+i \cdot P R F\right)
$$

Note that the value range of $f_{a}$ is $(-P R F / 2, P R F / 2)$ or $(0, P R F) . i$ represents the Doppler ambiguity index and the Doppler ambiguity number $I=i_{\max }-i_{\min }+1 . s\left(\tau, f_{a}+i \cdot P R F\right)$ denotes the equivalent unaliased Doppler spectrum of range bin $\tau$ at frequency $f_{a}+i \cdot P R F$. The azimuth MCSAR system signal with the addition of the noise signal and the use of vector symbols can be expressed as

$$
\mathbf{x}\left(\tau, f_{a}\right)=\sum_{i=i_{\min }}^{i_{\max }} \mathbf{a}_{i}\left(f_{a}\right) s\left(\tau, f_{a}+i \cdot P R F\right)+\mathbf{n}\left(\tau, f_{a}\right)
$$

where the $N \times 1$ vector $\mathbf{n}\left(\tau, f_{a}\right)$ represents the noise vector. The specific form of (9) is as follows

$$
\begin{gathered}
\mathbf{x}\left(\tau, f_{a}\right)=\left[x_{1}\left(\tau, f_{a}\right), \ldots, x_{N}\left(\tau, f_{a}\right)\right]^{T} \\
\mathbf{n}\left(\tau, f_{a}\right)=\left[n_{1}\left(\tau, f_{a}\right), \ldots, n_{N}\left(\tau, f_{a}\right)\right]^{T} \\
\mathbf{a}_{i}\left(f_{a}\right)=\left[a_{i}\left(f_{a}\right), \cdots, a_{N}\left(f_{a}\right)\right]^{T} \\
a_{i}\left(f_{a}\right)=\exp \left(j 2 \pi\left(f_{a}+i P R F\right) \frac{\Delta x_{i}}{v_{s}}\right), i=1, \cdots, N
\end{gathered}
$$

where the symbol $(\bullet)^{T}$ represents vector transpose. 


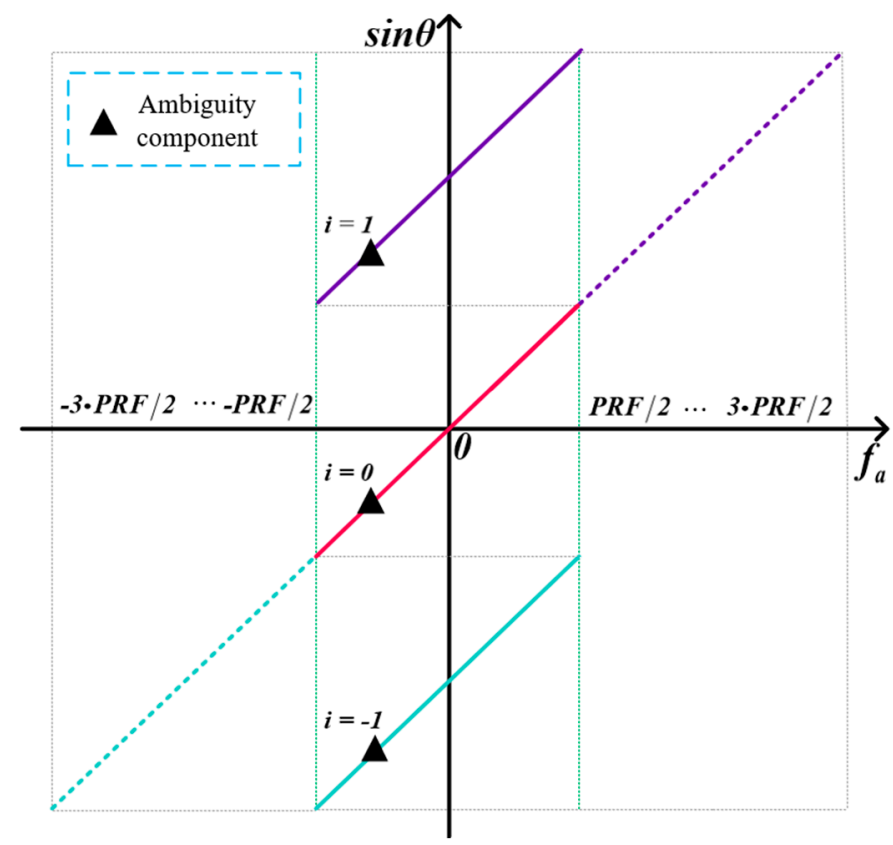

Figure 3. The relationship between Doppler frequency and spatial cone angle of azimuth multichannel SAR system.

\section{Related Work}

\subsection{The DBF Reconstruction Algorithm}

If the digital sampling signal satisfies the average Shannon-Nyquist sampling theorem, regardless of whether its spatial distribution is uniform, the signal spectrum can be recovered without ambiguity. Based on the above idea, Krieger et al. proposed the DBF algorithm to perform azimuth MCSAR signal-reconstruction [9]. The DBF algorithm obtains the equivalent single-channel uniformly sampled signal by reconstructing the azimuth MCSAR non-uniformly sampled signal. According to (3), the azimuth echo signal of the $m$ th channel can be expressed as

$$
x_{m}(\eta)=\sigma \cdot \exp \left[-j \frac{2 \pi \sqrt{R_{0}^{2}+\left(v_{s} \eta\right)^{2}}+\sqrt{R_{0}^{2}+\left(v_{s} \eta-\Delta x_{m}\right)^{2}}}{\lambda}\right]
$$

The echo signal of the reference channel can be written as

$$
x_{r e f}(\eta)=\sigma \cdot \exp \left(-j \frac{4 \pi}{\lambda} R_{0}\right) \cdot \exp \left(-j \frac{2 \pi v_{s}^{2} \eta^{2}}{\lambda R_{0}}\right)
$$

For $\Delta \eta_{m}=\Delta x_{m} /\left(2 v_{s}\right),(14)$ can be reformulated as

$$
x_{m}(\eta)=x_{r e f}\left(\eta-\Delta \eta_{m}\right) \cdot \exp \left(-j \frac{\pi \Delta x_{m}^{2}}{2 \lambda R_{0}}\right)
$$

We then transform (16) to the range-Doppler domain:

$$
\begin{aligned}
X_{m}\left(f_{a}\right) & =X_{r e f}\left(f_{a}\right) \cdot \exp \left(-j \frac{\pi \Delta x_{m}^{2}}{2 \lambda R_{0}}\right) \cdot \exp \left(-j 2 \pi f_{a} \Delta \eta_{m}\right) \\
& =X_{r e f}\left(f_{a}\right) \cdot H_{m}\left(f_{a}\right)
\end{aligned}
$$


Therefore, the filter function of each channel can be written as

$$
H_{m}\left(f_{a}\right)=\exp \left(-j \frac{\pi \Delta x_{m}^{2}}{2 \lambda R_{0}}\right) \cdot \exp \left(-j \frac{\pi \Delta x_{m}}{v_{s}} f_{a}\right)
$$

The filter function matrix can be expressed as

$$
\mathbf{H}_{N \times N}\left(f_{a}\right)=\left[\begin{array}{ccc}
H_{1}\left(f_{a}\right) & \cdots & H_{N}\left(f_{a}\right) \\
\vdots & \ddots & \vdots \\
H_{1}\left(f_{a}+(N-1) P R F\right) & \cdots & H_{N}\left(f_{a}+(N-1) P R F\right)
\end{array}\right]
$$

According to the literature [9], the signal of each channel is reconstructed by its own filters and then superimposed. Finally, an unambiguous single-channel signal spectrum can be obtained. The relationship between the reconstruction filter matrix $\mathbf{P}\left(f_{a}\right)$ and $\mathbf{H}\left(f_{a}\right)$ is

$$
\mathbf{P}_{N \times N}\left(f_{a}\right)=\left[\begin{array}{ccc}
P_{11}\left(f_{a}\right) & \cdots & P_{1 N}\left(f_{a}+(N-1) \cdot P R F\right) \\
\vdots & \ddots & \vdots \\
P_{N 1}\left(f_{a}\right) & \cdots & P_{N N}\left(f_{a}+(N-1) \cdot P R F\right)
\end{array}\right]=\mathbf{H}_{N \times N}^{-1}\left(f_{a}\right)
$$

For filter $\mathbf{H}\left(f_{a}\right)$, if the sampling positions coincide, $\mathbf{H}\left(f_{a}\right)$ is irreversible, and the DBF algorithm is invalid. According to Formula (20), the filter function of each channel in the azimuth can be obtained. Each row of $\mathbf{P}_{N \times N}\left(f_{a}\right)$ represents the reconstruction filter $P_{n}\left(f_{a}\right)$ of each channel. $P_{n}\left(f_{a}\right)$ is composed of $N$ filters $P_{n j}\left(f_{a}\right)(j=1, \ldots, N)$. The $N$ filters divide the whole frequency band $I_{S}=\left[-\frac{N \cdot P R F}{2}, \frac{N \cdot P R F}{2}\right]$ into $N$ sub-bands, and the center frequency of each sub-band is $f_{0, j}=\left(j-\frac{N+1}{2}\right) \cdot P R F$, the corresponding sub-band range is

$$
I_{j}=\left[-\frac{N \cdot P R F}{2}+(j-1) \cdot P R F,-\frac{N \cdot P R F}{2}+j \cdot P R F\right]
$$

Moreover, the DBF algorithm is implemented without any prior knowledge of the covariance matrix and can be implemented by matrix inversion. At the same time, the DBF algorithm is computationally efficient and does not need to know the unambiguous Doppler spectrum and the azimuth antenna pattern. However, the DBF algorithm does not consider the additive noise and was derived for a band-limited signal. In the ideally configured MCSAR system, the DBF algorithm can perfectly reconstruct the signal with low ambiguities and noise levels. However, when the PRF is close to the coinciding with $P R F$, the SNR of the DBF algorithm will be severely reduced.

\subsection{The IDBF Reconstruction Algorithm}

To overcome the shortcoming of Kriger's DBF algorithm, wherein the SNR is reduced in the case of highly non-uniform sampling, Liu et al. proposed the IDBF algorithm [21]. The IDBF algorithm consists of two procedures: the first realizes an operation of AIS based on the equivalent sampling interval and ERDB. The second implements Doppler spectrum weighting based on the maximum-to-minimum ratio of the noise spectrum.

The IDBF algorithm starts from the perspective of the SNR scale factor and improves the performance of the DBF algorithm by reducing the value of the SNR scale factor. Liu et al. proved that the value of the SNR scale factor could be reduced by reducing the dimensionality of the MCSAR signal. Additionally, the IDBF algorithm determines which Doppler components need to be restored and which components need to be ignored by defining AIS (see Table I in [21]). Having constructed the AIS, a new channel-transfer function matrix can be constructed (see Equation (38) [21]). According to the $Q R$-factorization version of the DBF algorithm (see Table II [21]), the two-dimensional spectrum reconstruction can be completed. To overcome the problem of the occurrence of discontinuities for the noise spectrum after applying the new version of the DBF algorithm, the IDBF algorithm 
considers adopting a weighting strategy. However, the cost of weighting is the loss of azimuth resolution.

The key point of the IDBF algorithm is to calculate ERDB and AIS so as to obtain ambiguous information but at the cost of increasing AASR and decreasing the azimuth resolution.

\section{Proposed Approach}

\subsection{Problem Formulation}

The DBF algorithm ignores the influence of noise when solving the filter coefficients, but the noise always exists. Therefore, the signal model shown in (9) will be considered in this paper. SNR scale factor characterizes the influence of the digital reconstruction filter network on the SNR. In the literature [25], it was proven that the output noise power of the DBF algorithm is significantly amplified in the case of highly non-uniform sampling, and the amplification ratio is as high as $15 \mathrm{~dB}$ [31]. The SNR scale factor is defined as the SNR of the input signal to the SNR of the reconstruction filter output signal. It can also be understood as the ratio of the noise amplification factor to the signal amplification factor [24]. To facilitate subsequent discussion, the definition of the SNR scale factor in [25] is repeated in this section. The specific expression of the SNR scale factor $\Phi_{b f}$ is as follows:

$$
\Phi_{b f}=\frac{S N R_{\text {in }}}{S N R_{\text {out }}}=\frac{A_{n}}{A_{S}}
$$

where $S N R_{\text {in }}$ represents the SNR of the input signal, $S N R_{\text {out }}$ represents the SNR of the reconstruction filter output signal, $A_{n}$ represents the noise amplification factor, and $A_{s}$ represents the signal amplification factor. Since the reconstruction filter system is linear and only considers additive white noise, the signal power and noise power can be analyzed separately.

The input power of the signal is defined as the average energy of the signal input to the digital reconstruction filter network within the equivalent reconstruction bandwidth $B_{a}$. Combined with the multi-channel SAR echo signal model, the input signal power $P_{s i}$ can be written as

$$
P_{s i}=\boldsymbol{E}\left[\sum_{i=i_{\min }}^{i_{\max }} s^{H}\left(\tau, f_{a}+i P R F\right) \mathbf{a}_{i}^{H} \cdot \sum_{i=i_{\min }}^{i_{\max }} s\left(\tau, f_{a}+i P R F\right) \mathbf{a}_{i}\right]
$$

where $\boldsymbol{E}(\bullet)$ means to find the mean value in the range of $B_{a},(\bullet)^{H}$ represents conjugate transpose. Taking into account the incoherence of different signal components, that is, $\boldsymbol{E}\left[x\left(\tau, f_{a}+i P R F\right) x\left(\tau, f_{a}+j P R F\right)\right]=0(i \neq j),(23)$ can be simplified as

$$
\begin{aligned}
P_{s i} & =\boldsymbol{E}\left[\sum_{i=i_{\min }}^{i_{\max }} s^{H}\left(\tau, f_{a}+i P R F\right) \mathbf{a}_{i}^{H} \mathbf{a}_{i} s\left(\tau, f_{a}+i P R F\right)\right] \\
& =\boldsymbol{E}\left[M \sum_{i=i_{\text {min }}}^{i_{\text {max }}} s^{H}\left(\tau, f_{a}+i P R F\right) s\left(\tau, f_{a}+i P R F\right)\right]
\end{aligned}
$$

The reconstructed Doppler frequency $f_{b}=f_{a}+i \cdot P R F$, the range of $f_{b}$ is $\left[-B_{a} / 2, B_{a} / 2\right]$. Since the value range of $f_{a}$ is $[0, P R F]$ or $[-P R F / 2, P R F / 2]$, the input power $P_{s i}$ of the filter network can be rewritten as

$$
P_{s i}=M \cdot E\left[s^{H}\left(\tau, f_{b}\right) s\left(\tau, f_{b}\right) \operatorname{rect}\left(\frac{f_{b}}{B_{a}}\right)\right]
$$


The meaning of the window function $\operatorname{rect}\left(\frac{f_{b}}{B_{a}}\right)$ is

$$
\operatorname{rect}\left(\frac{f_{b}}{B_{a}}\right)=\left\{\begin{array}{c}
1,\left|\frac{f_{b}}{B_{a}}\right| \leq 1 / 2 \\
0,\left|\frac{f_{b}}{B_{a}}\right|>1 / 2
\end{array}\right.
$$

We can assume that the noise in the raw data is the additive white noise (only Gaussian white noise). Then, $S N R_{\text {in }}$ can be expressed as

$$
S N R_{i n}=\frac{P_{s i}}{\sigma_{n}^{2}}=\frac{M \cdot E\left[S^{H}\left(\tau, f_{b}\right) s\left(\tau, f_{b}\right) \operatorname{rect}\left(\frac{f_{b}}{B_{a}}\right)\right]}{\sigma_{n}^{2}}
$$

where $\sigma_{n}^{2}$ represents the noise power of the input filter. The power of the output signal can be expressed as

$$
\begin{aligned}
P_{s o} & =\boldsymbol{E}\left[\left\|\boldsymbol{\omega}_{i}^{H} \mathbf{a}_{i}\right\|^{2} s\left(\tau, f_{b}\right) \operatorname{rect}\left(\frac{f_{b}}{B_{a}}\right)\right] \\
& =\boldsymbol{E}\left[\boldsymbol{\omega}^{H}\left(f_{b}\right) \mathbf{a}_{i} s\left(\tau, f_{b}\right) s^{H}\left(\tau, f_{b}\right) \mathbf{a}_{i}^{H} \boldsymbol{\omega}_{i} \operatorname{rect}\left(\frac{f_{b}}{B_{a}}\right)\right] \\
& =\boldsymbol{E}\left[s^{H}\left(\tau, f_{b}\right) s\left(\tau, f_{b}\right) \operatorname{rect}\left(\frac{f_{b}}{B_{a}}\right)\right]
\end{aligned}
$$

where $\boldsymbol{\omega}_{i}$ represents the filter coefficient and $\boldsymbol{\omega}\left(f_{b}\right)=\boldsymbol{\omega}_{i}$ (see [31], Equations (2)-(54)). After the azimuth ambiguity suppression, the output noise power $P_{n o}$ of the reconstruction filter network can be expressed as

$$
P_{n o}=\sigma_{n}^{2} \boldsymbol{E}\left[\boldsymbol{\omega}_{i}^{H} \boldsymbol{\omega}_{i} \operatorname{rect}\left(\frac{f_{b}}{B_{a}}\right)\right]
$$

Combining Formulas (28) and (29), the $S N R_{\text {out }}$ of the reconstruction filter network can be calculated as

$$
S N R_{\text {out }}=\frac{P_{\text {so }}}{P_{n o}}=\frac{\boldsymbol{E}\left[s^{H}\left(\tau, f_{b}\right) s\left(\tau, f_{b}\right) \operatorname{rect}\left(\frac{f_{b}}{B_{a}}\right)\right]}{\sigma_{n}^{2} \boldsymbol{E}\left[\boldsymbol{\omega}_{i}^{H} \boldsymbol{\omega}_{i} \operatorname{rect}\left(\frac{f_{b}}{B_{a}}\right)\right]}
$$

According to the definition of the SNR scale factor, combining (22), (27), and (30), we can obtain

$$
\Phi_{b f}=M \cdot \boldsymbol{E}\left[\boldsymbol{\omega}^{H}\left(f_{b}\right) \boldsymbol{\omega}\left(f_{b}\right) \operatorname{rect}\left(\frac{f_{b}}{B_{a}}\right)\right]
$$

For the DBF algorithm, the ambiguity number of each Doppler unit is $M$. Substituting $\boldsymbol{\omega}_{i}$ into (31), we can obtain

$$
\Phi_{b f}=M \cdot \frac{1}{M} E\left[\sum_{i} \boldsymbol{\omega}_{i}^{H} \boldsymbol{\omega}_{i}\right]=\operatorname{trace}\left[\left(\mathbf{P}^{H}\left(f_{b}\right) \mathbf{P}\left(f_{b}\right)\right)^{-1}\right]=\frac{1}{\lambda_{1}^{2}}+\frac{1}{\lambda_{2}^{2}}+\cdots+\frac{1}{\lambda_{N}^{2}}
$$

where trace $[\bullet]$ represents finding the trace of the square matrix and $\lambda_{m}$ means the singular value of the square matrix $\mathbf{P}\left(f_{b}\right)$.

It is worth noting that (32) is similar in form to the equation derived in [25] (see Equation (36) in [25]). When the PRF of the azimuth multi-channel SAR system satisfies the uniform sampling condition, $\lambda_{1}^{2}=\lambda_{2}^{2}=\cdots=\lambda_{N}^{2}=N, \Phi_{b f}=1$ is the minimum value. It can be seen from (32) that as the degree of non-uniform azimuth sampling increases, the noise power of the signal is gradually amplified, and the SNR of the DBF algorithm is greatly reduced. When redundant sampling occurs, the DBF algorithm stops working. This 
occurs because the matrix $\mathbf{H}\left(f_{a}\right)$ is singular and noninvertible. For the redundant sampling scenario, the value of $\Phi_{b f}$ is infinite, which means that the noise power is amplified to infinity, and the DBF algorithm cannot work as a result.

\subsection{The Proposed Method}

As discussed in Section 3, in the case of highly non-uniform sampling, the SNR of the DBF algorithm would be severely reduced. To solve the above problem, this section proposes the TSPT algorithm based on the idea of sparse uniform resampling. The TSPT algorithm reconstruction process consists of two steps in Figure 4. The first is to project the azimuth multi-channel SAR signal obliquely to $\mathcal{T}$ [26]. It can recover a consistent approximation of $x_{m}\left(\tau, f_{a}\right)$ in $\mathcal{T}$, denoted $x_{\mathcal{T}}$. In this case, consistency means that the original sample will produce $\mathbf{b}$. The second projection is to orthogonally project the result of the first projection onto $\mathcal{A}$. This projection restores the signal closest to the signal $x_{\mathcal{T}}$ in $\mathcal{A}$. Using a digital LSI filter can achieve the secondary projection and generate evenly spaced signal values. The introduction of intermediate subspace can achieve low computational complexity and keep the approximation error.

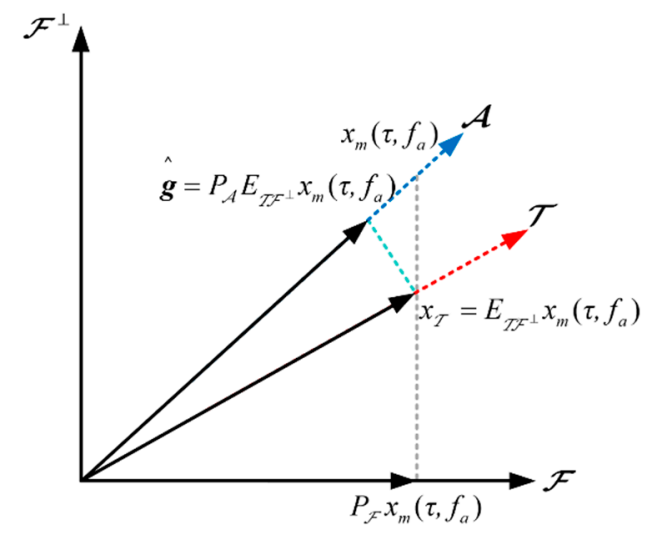

Figure 4. Signal projection geometry.

Introducing the intermediate subspace $\mathcal{T} \in \mathcal{H}$ (Hilbert space), which is spanned by the set $\left\{\mathbf{t}_{n}\right\}$, including the integer translation of the compact support function $t(k)$, i.e.,

$$
\mathbf{t}_{n}(k)=t(k / \Delta-n)
$$

Using $x_{\mathcal{T}}=\mathcal{T} \mathbf{c}$ to represent the consistent reconstruction of $x_{m}\left(\tau, f_{a}\right)$ in $\mathcal{T}$, which is given by the oblique projection of $\mathcal{F}^{\perp}$ to $\mathcal{T}$, i.e.,

$$
x_{\mathcal{T}}=\mathcal{T} \mathbf{c}=\mathcal{T}\left(F^{*} \mathcal{T}\right)^{\dagger} \mathbf{b}=\mathcal{T}\left(F^{*} \mathcal{T}\right)^{\dagger} F^{*} x_{m}\left(\tau, f_{a}\right)=E_{\mathcal{T} \mathcal{F}^{\perp}} x_{m}\left(\tau, f_{a}\right)
$$

where $(\bullet)^{\dagger}$ represents Moore-Penrose pseudoinverse, and when $\left(F^{*} \mathcal{T}\right)^{\dagger}$ is invertible, $\left(F^{*} \mathcal{T}\right)^{\dagger}=\left(F^{*} \mathcal{T}\right)^{-1} . F$ corresponds to a set of vectors $\left\{\mathbf{f}_{m}\right\}$; these vectors form the subspace $\mathcal{F}$ and form the Riesz basis; $F^{*}$ is the adjoint of $F$. Using this notation, the vector $\mathbf{b}$ can be given by $\mathbf{b}=F^{*} x_{m}\left(\tau, f_{a}\right)$, where $b[m]=\mathbf{f}_{m}, x_{m}\left(\tau, f_{a}\right)$.

$\mathrm{c}$ is obtained by formalizing and solving the equation relating the non-uniform sample $\mathbf{b}$ to the coefficient vector $\mathbf{c}$, i.e.,

$$
\mathbf{b}=F^{*} \mathcal{T} \mathbf{c}
$$

Using $\mathbf{c}$ to define $x_{\mathcal{T}}$, and given the knowledge that $x_{m}\left(\tau, f_{a}\right) \in \mathcal{A}$, the next projection is to project $x_{\mathcal{T}}$ to $\mathcal{A}$, and in the sense of $L_{2}$, the best projection scheme is $x_{\mathcal{T}}$ orthogonal projection to $\mathcal{A}$, denoted $P_{\mathcal{A}}$. Since both $\mathcal{A}$ and $\mathcal{T}$ are shift-invariant subspaces, $P_{\mathcal{A}} x_{\mathcal{T}}$ can be calculated by using LSI filters. According to the geometric interpretation of signal 
projection given in Figure 4, the two projection sequences of the proposed method can be expressed as

$$
\hat{g}=A\left(A^{*} A\right)^{\dagger} A^{*} \mathcal{T}\left(F^{*} \mathcal{T}\right)^{\dagger} F^{*} x_{m}\left(\tau, f_{a}\right)
$$

where $P_{\mathcal{A}}=A\left(A^{*} A\right)^{\dagger} A^{*}$ and $E_{\mathcal{T} \mathcal{F} \perp}=\mathcal{T}\left(F^{*} \mathcal{T}\right)^{\dagger} F^{*}$.

We divide the above formula into two steps for calculation. First, for a given azimuth multi-channel SAR signal $\mathbf{b}$, in the sense of least squares, $\mathbf{c}$ is calculated by (35), i.e.,

$$
\mathbf{c}=\left(F^{*} \mathcal{T}\right)^{\dagger} \mathbf{b}
$$

Subsequently, we use c to calculate the coefficients $\mathbf{d}$, i.e.,

$$
\mathbf{d}=\left(A^{*} A\right)^{\dagger}\left(A^{*} \mathcal{T}\right) \mathbf{c}
$$

The reconstruction process is given by $\hat{g}=A \mathbf{d}$.

To calculate $c$, let us explicitly formalize $x_{m}\left(\tau, f_{a}\right)$ as defined in (8):

$$
b[m]=\sum_{n} c[n] t\left(k_{m}-k_{n}\right)
$$

where $k_{m}$ is the non-uniformly sampling points in the frequency domain. On account of the compact support of $t$, only a few coefficients $c[n]$ in (39) can calculate the value of $b[m]$. Therefore, using a $M \times N$ sparse matrix $\boldsymbol{\Phi}$ represents the sparse relation between the coefficient vectors $\mathbf{b}$ and $\mathbf{c}$. The elements in $\boldsymbol{\Phi}$ can be expressed as

$$
\{\boldsymbol{\Phi}\}_{m, n}=\left\{F^{*} \mathcal{T}\right\}_{m, n}
$$

To find c, a weighted regularized least-squares problem is formulated

$$
\mathbf{c}=\operatorname{argmin}\left\|\bar{\Gamma}\left(\mathbf{b}-\mathbf{\Phi c}^{\prime}\right)\right\|^{2}+\rho\left\|\mathbf{c}^{\prime}\right\|^{2}
$$

where $\|\cdot\|$ represents the norm of $\mathbf{c}, \rho$ is a Tikhonov regularization parameter, $\Gamma$ is an $M \times M$ diagonal weighting matrix, and $\bar{\Gamma}=\Gamma^{\frac{1}{2}}$.

To calculate (41), the Hachtel-augmented matrix method gives a useful sparse preserving formula for normal equations. This formulation is extended by us to accommodate for the weights and the regularization. By defining the remainder $\mathbf{r}=\boldsymbol{\Gamma}(\mathbf{b}-\boldsymbol{\Phi} \mathbf{c})$, after deriving (41), it can be reformulated as

$$
\left(\begin{array}{c}
- \\
\boldsymbol{\Gamma} \mathbf{b} \\
\mathbf{0}
\end{array}\right)=\left(\begin{array}{cc}
\mathbf{I} & \bar{\Gamma} \boldsymbol{\Phi} \\
-T & \\
\boldsymbol{\Phi}^{T} \boldsymbol{\Gamma} & -\rho \mathbf{I}
\end{array}\right)\left(\begin{array}{l}
\mathbf{r} \\
\mathbf{c}
\end{array}\right)=\mathbf{\Psi}\left(\begin{array}{l}
\mathbf{r} \\
\mathbf{c}
\end{array}\right)
$$

where $\boldsymbol{\Psi}$ maintains the sparsity of $\boldsymbol{\Phi}$.

Using the direct inversion method to calculate $\mathbf{\Psi}$, the complexity is $O\left((M * N)^{3}\right)$, which is prohibited by the computer. Moreover, even if $\Psi^{-1}$ were known, it would still require $O\left((M * N)^{2}\right)$ operations to compute $\mathbf{c}$ from $\mathbf{b}$ in (42). To solve the matrix $\mathbf{\Psi}$ with low computational complexity, the sparse equation solver is used to calculate the LU factor of $\boldsymbol{\Psi}$. The process of solving (42) is divided into two steps: first, LU factorization of $\boldsymbol{\Psi}$ using the sparse solver package UMFPACK [32], i.e.,

$$
\mathbf{P}\left(\mathbf{R}^{-1} \mathbf{\Psi}\right) \mathbf{Q}=\mathbf{L U}
$$


where $\mathbf{P}$ and $\mathbf{Q}$ are permutation matrices; $\mathbf{R}$ is a diagonal scaling matrix, which helps to achieve a sparser and more stable decomposition; and $\mathbf{L}$ and $\mathbf{U}$ are upper and lower triangular matrices, respectively [32]. For more calculation details, please refer to [33]. Secondly, for a given non-uniformly sampled azimuth MCSAR signal $\mathbf{b}$, the calculation of c using $\mathbf{L}$ and $\mathbf{U}$ is completed through forward substitution and backward elimination.

The vector $\mathbf{c}$ can be calculated by the above, and then the vector $\mathbf{d}$ in (38) is calculated. Since both $\mathcal{T}$ and $\mathcal{A}$ correspond to the integer shift of the kernel function, $\mathbf{d}$ can be calculated efficiently by an LSI filter [34], i.e.,

$$
H_{L S I}\left(e^{j \omega}\right)=\frac{R_{\mathcal{A T}}\left(e^{j \omega}\right)}{R_{\mathcal{A A}}\left(e^{j \omega}\right)}
$$

where

$$
R_{\mathcal{A T}}\left(e^{j \omega}\right)=\mathbb{F}\left\{r_{a t}[n]\right\}=\sum_{n \in \mathbb{Z}} r_{a t}[n] e^{-j \omega n}
$$

is the Fourier transformation of the sampled correlation sequence, and $\mathbb{F}\{\bullet\}$ represents the Fourier transformation. $r_{a t}[n]$ can be expressed as

$$
r_{a t}[n]=\langle a(k), t(k+n \Delta)\rangle=\int_{-\infty}^{\infty} a(k), t(k+n \Delta) d k
$$

Combined with (45), (46) can be rewritten as

$$
R_{\mathcal{A}}\left(e^{j \omega}\right)=\frac{1}{\Delta} \sum_{n \in \mathbb{Z}} \overline{A\left(\frac{\omega}{\Delta}-\frac{2 \pi n}{\Delta}\right)} \mathcal{T}\left(\frac{\omega}{\Delta}-\frac{2 \pi n}{\Delta}\right)
$$

where $\mathcal{T}(\omega)$ is the Fourier transform of $t(k / \Delta)$, i.e.,

$$
\mathcal{T}(\omega)=\mathbb{F}\{t(k / \Delta)\}=\int_{-\infty}^{\infty} t(k / \Delta) e^{-j \omega k} d k
$$

The derivation process of $R_{\mathcal{A A}}\left(e^{j \omega}\right)$ and $A(\omega)$ is similar to the derivation process of $R_{\mathcal{A T}}\left(e^{j \omega}\right)$ and $\mathcal{T}(\omega)$. The coefficients of the vector $\mathbf{d}$ can be expressed as

$$
d[n]=\sum_{k \in \mathbb{Z}} c[n] h_{L S I}[n-k]
$$

According to the convolution nature of the Fourier transform, the above formula can be rewritten as

$$
D\left(e^{j \omega}\right)=H_{L S I}\left(e^{j \omega}\right) C\left(e^{j \omega}\right)
$$

where $C\left(e^{j \omega}\right)$ and $D\left(e^{j \omega}\right)$ are the Fourier transform of $\mathbf{c}$ and $\mathbf{d}, H_{L S I}\left(e^{j \omega}\right)=\mathbb{F}\left\{h_{L S I}[n]\right\}$. After calculating $\mathbf{d}$, a uniformly sampled signal can be obtained. After the traditional SAR chirp scaling (CS) algorithm, an image without ambiguity can be obtained. Since the proposed algorithm is actually performed in the frequency domain, it is relatively simple to use the CS algorithm.

To summarize, the TSPT algorithm solves the sparse equation of (42) by LU decomposition for a given non-uniformly sampled multi-channel SAR signal $\mathbf{b}$. Then, using the digital correction filter $H_{L S I}$ of formula (44) to filter the solved result, we store the finally calculated uniformly sampled samples in the vector $\mathbf{d}$. The flow of the proposed algorithm can be seen in Figure 5. To introduce the proposed algorithm in more detail, Algorithm 1 shows the pseudocode of the TSPT algorithm. 

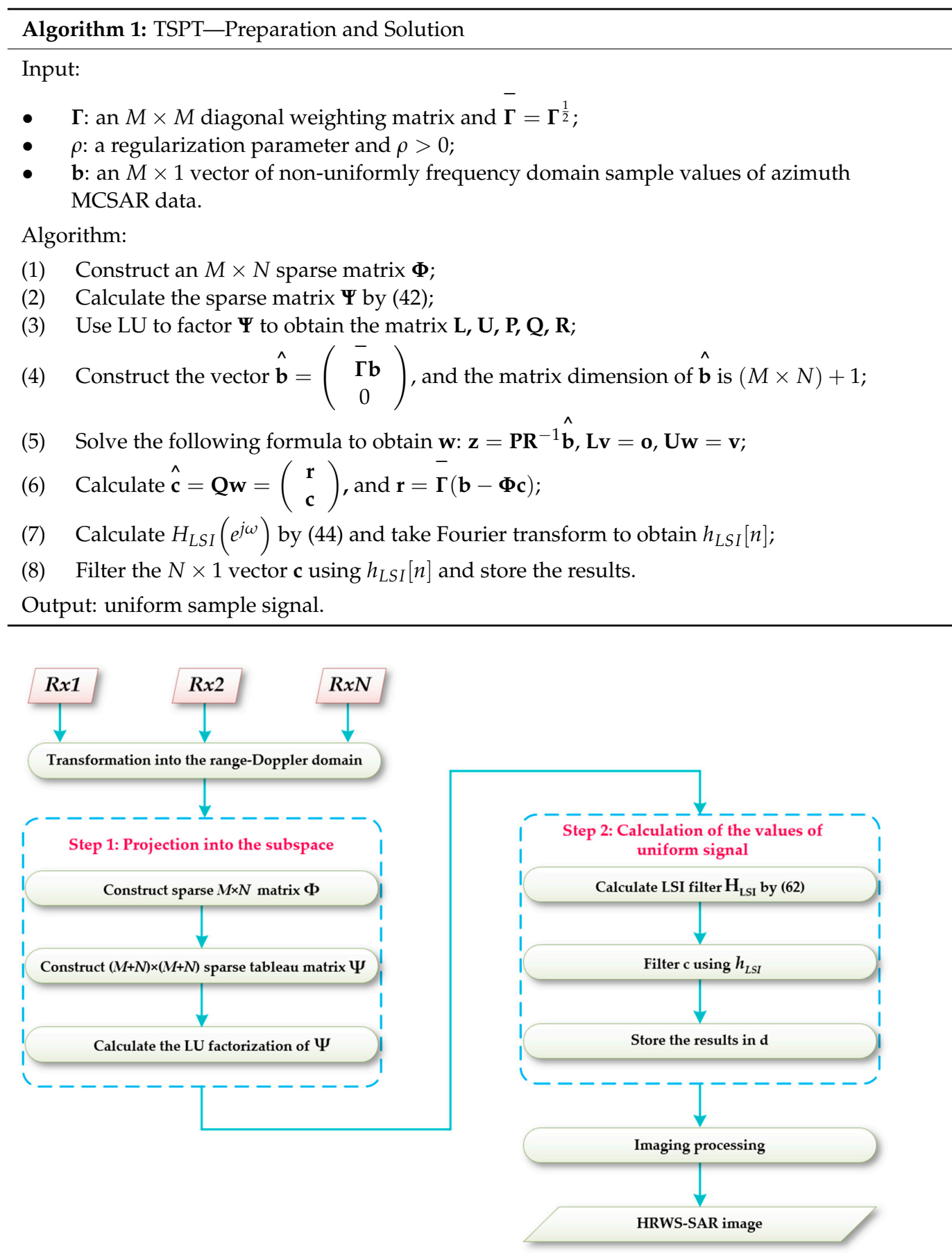

Figure 5. Block diagram of the TSPT algorithm.

\section{Experiment Results}

\subsection{Simulation Results}

In this section, a simulation experiment is carried out to evaluate the performance of the proposed reconstruction algorithm. At the same time, the comparison experiment with the DBF algorithm, the TSPT algorithm, and the IDBF algorithm is completed. Azimuth MCSAR system simulation parameters are shown in Table 1 . In the simulation, the system sets three receiving channels uniformly along the azimuth and sets the middle channel as the reference channel to transmit and receive signals. According to Formula (1), the PRF value of uniform sampling can be calculated as $1495 \mathrm{~Hz}$. When the azimuth sampling rate of the system deviates from the PRF value of uniform sampling, the azimuth MCSAR 
system signal can be regarded as non-uniform sampling. The value of $P R F$ of the simulation experiment is set to $1100 \mathrm{~Hz}$.

Table 1. Simulation parameters.

\begin{tabular}{cc}
\hline Parameter & Value \\
\hline Carrier Wavelength & $0.03 \mathrm{~m}$ \\
Doppler Band Width & $3737.4 \mathrm{~Hz}$ \\
Channel Number & 3 \\
Platform Velocity & $7474.8 \mathrm{~m} / \mathrm{s}$ \\
Adjacent Channel Interval & $3.3333 \mathrm{~m}$ \\
Slant Range & $890 \mathrm{~km}$ \\
Uniform $P R F$ & $1495 \mathrm{~Hz}$ \\
\hline
\end{tabular}

Due to the working frequency of the azimuth MCSAR system deviating from the ideal $P R F$, the echo signal will appear as Doppler spectrum aliasing. The imaging result will appear as ambiguous without reconstruction. Obviously, azimuth ambiguity occurs in each channel of MCSAR signal during azimuth under-sampling, resulting in virtual targets. In this section, point target contrast experiments and multiple-point targets simulation experiment are carried out. We added independent white noise to the data so that the SNR of the image domain after signal-reconstruction is about $50 \mathrm{~dB}$. It can be clearly seen from Figure 6 that in the case of signal with noise, the imaging results of the TSPT algorithm are significantly better than the DBF algorithm and the IDBF algorithm. As shown in Figure 7, according to the imaging result of nine point targets, the TSPT algorithm has good reconstruction performance in the case of multiple point targets. In Figure 7, the left half is the imaging result of nine points, and it can be seen that there is no azimuth ambiguity after the reconstruction of the azimuth signal by the proposed method. The right half of Figure 7 shows the contour map, azimuth profile, and range profile of the edge point target. In summary, the accuracy of the proposed reconstruction algorithm can meet the requirements of SAR imaging and also lays a foundation for the processing of real data.

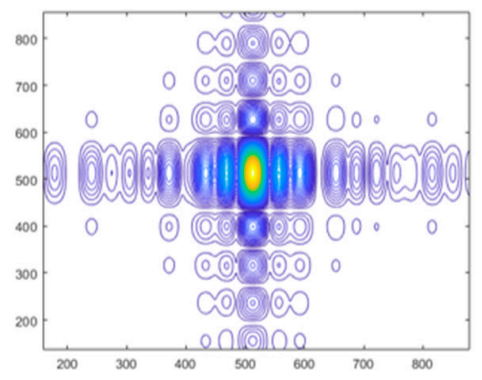

(a)

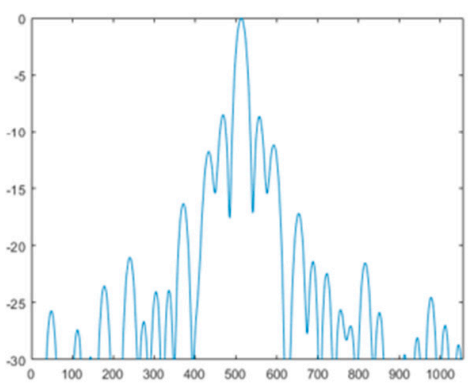

(d)

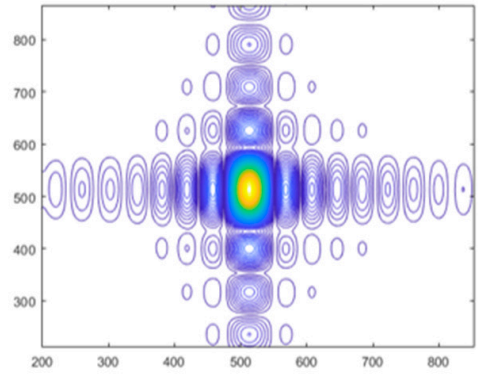

(b)

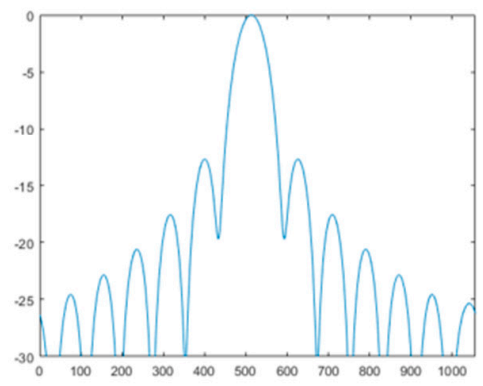

(e)

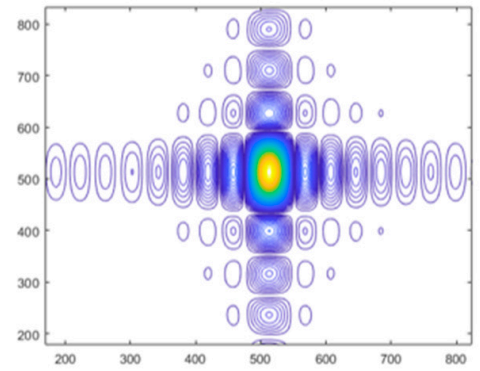

(c)

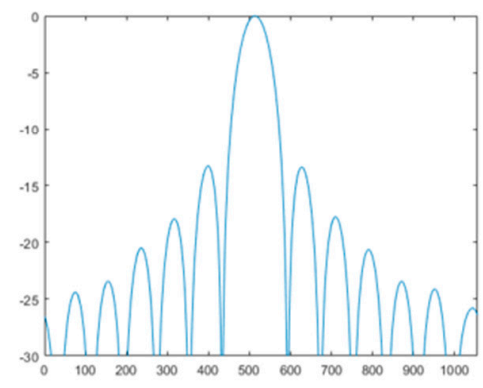

(f)

Figure 6. Point target simulation. (a) DBF. (b) IDBF. (c) TSPT. (d) Azimuth profile corresponding to (a). (e) Azimuth profile corresponding to (b). (f) Azimuth profile corresponding to (c). 

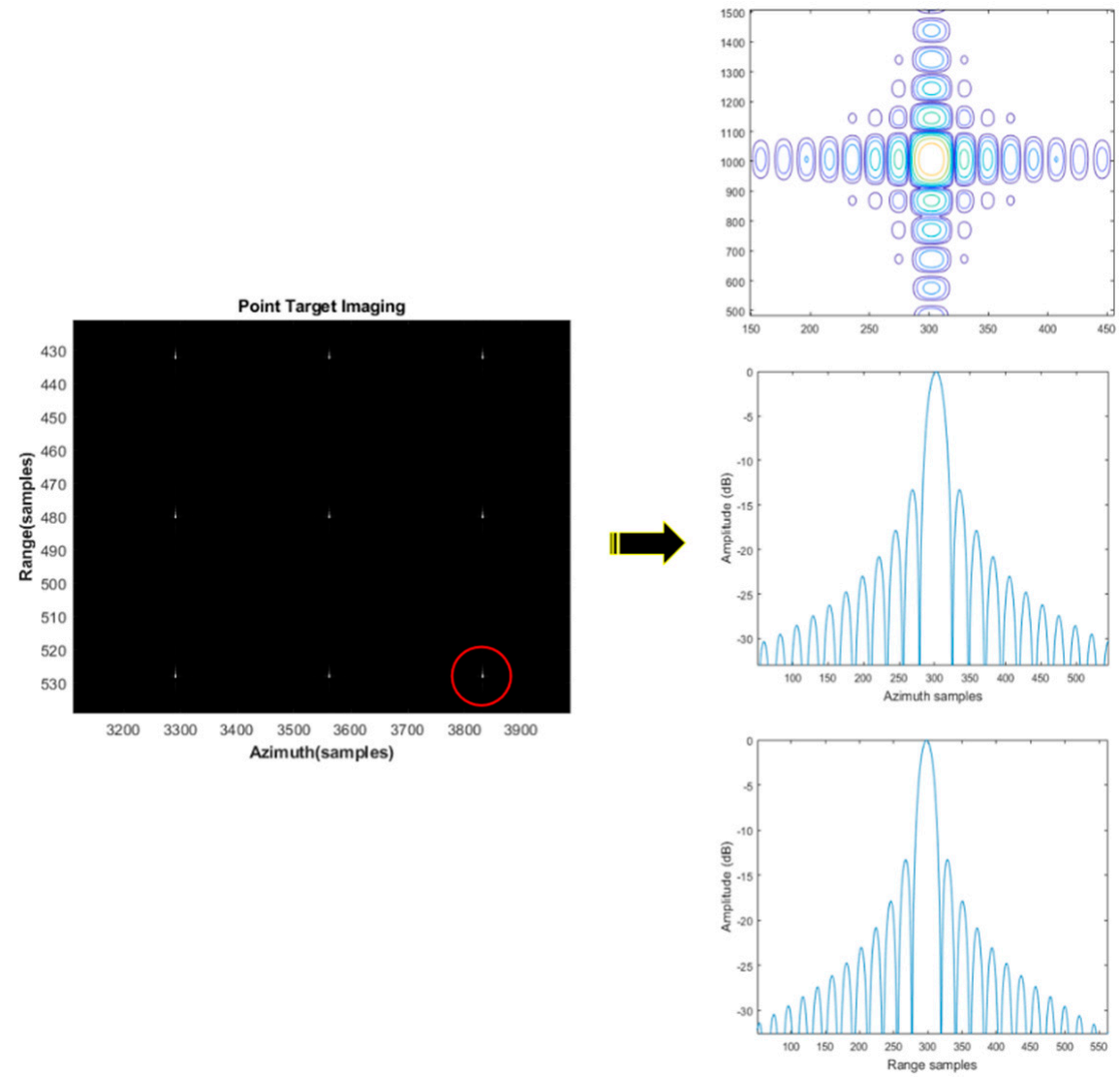

Figure 7. (Left) Mutiple point targets imaging. (Right) Edge point target contour map, azimuth profile, and range profile.

To verify the performance of the proposed method, this section gives a comparison experiment on the SNR and AASR of the DBF algorithm, the TSPT algorithm, and the IDBF algorithm. For the calculation method of the SNR of the TSPT algorithm, refer to [26], and for the calculation method of AASR, refer to [25,35]. For each PRF in the interval of [1495-2803] Hz, we generate azimuth three-channel SAR data according to the parameters shown in Algorithm 1. Then, the DBF algorithm, the TSPT algorithm, and the IDBF algorithm were used to process the data of the three channels. Figure 8 shows the SNR and AASR of the three methods calculated for different $P R F$ values. It can be seen from Figure 8a that the SNR of the DBF algorithm, TSPT algorithm, and IDBF algorithm are consistent under uniform sampling. It can be seen that when the system PRF deviates from the uniformly sampled $P R F$, the SNR of the DBF algorithm will drop sharply. For PRFs close to coinciding with PRF, the DBF algorithm has a quite low SNR compared with the TSPT algorithm and the IDBF algorithm.

To observe the inhibitory effect of the three methods on the AASR after the azimuth reconstruction, the AASR of the DBF algorithm, the TSPT algorithm, and the IDBF algorithm was calculated. To view the suppression effects of the different methods, AASR after azimuth reconstruction by the DBF algorithm, the TSPT algorithm, and the IDBF algorithm, respectively, are depicted in Figure 8b. Examining Figure 8b, it can be seen that the AASR of the TSPT algorithm is higher than those of the other two algorithms.

Furthermore, the comparison results in this section validate such an argument that the proposed algorithm is a relatively reasonable candidate algorithm. The TSPT algorithm can solve the problem of spectrum reconstruction of non-uniformly sampled azimuth MCSAR signals. The proposed algorithm can not only ensure high SNR but also suppress azimuth ambiguity. 


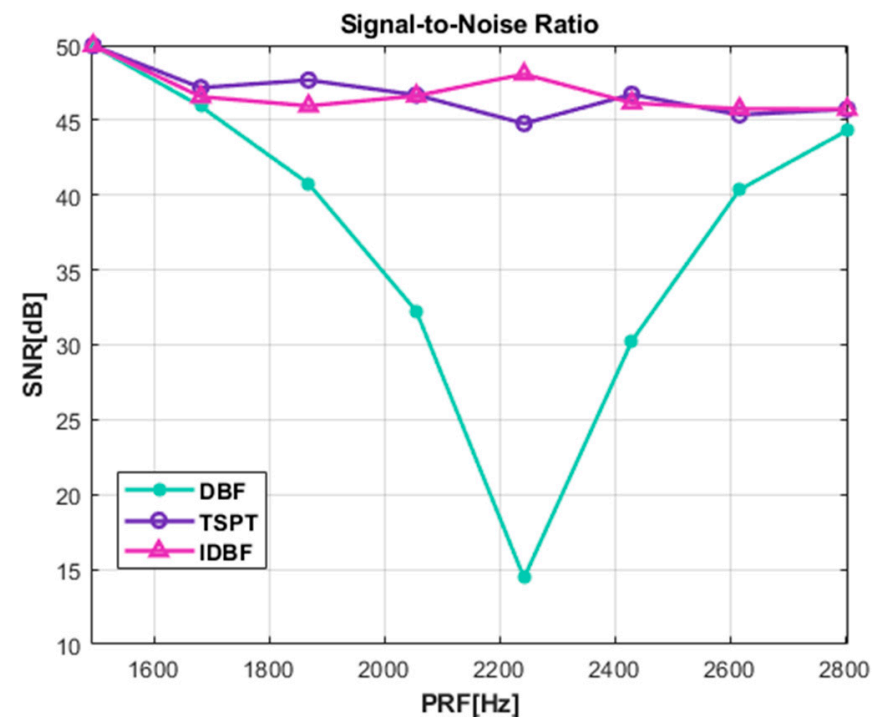

(a)

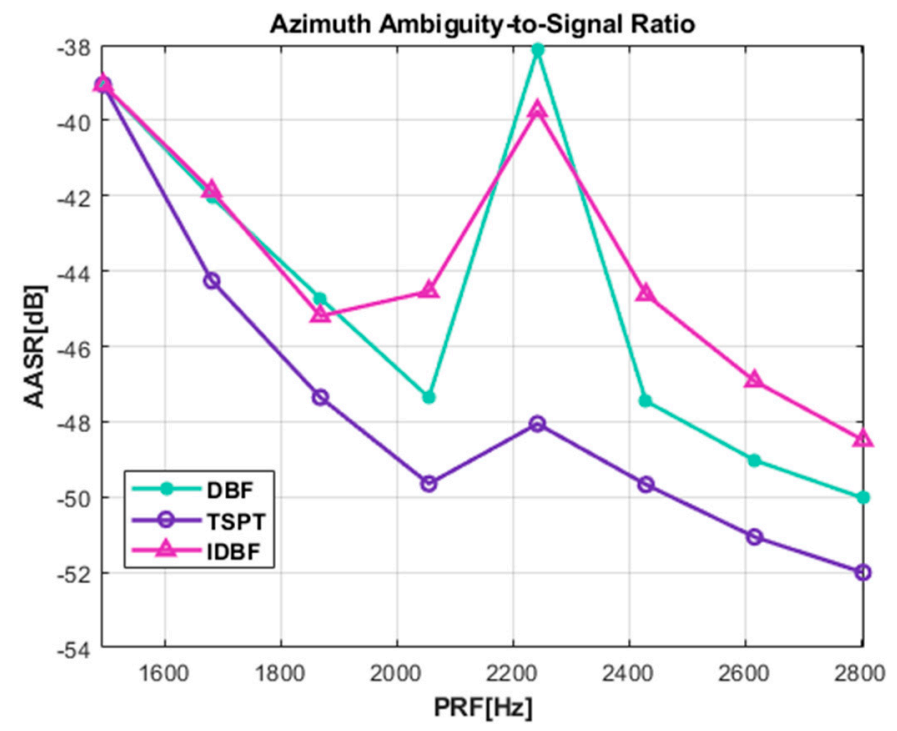

(b)

Figure 8. Performance of the different reconstruction methods against varying PRF. (a) SNR. (b) AASR.

\subsection{MCSAR Real Data Processing}

Aiming at better showing the performance of the proposed algorithm, this section has carried out the MCSAR real data processing. The real data used are the stripe mode of the L-band airborne azimuth MCSAR system, and the real data parameters are shown in Table 2. It can be seen from the parameters in Table 2 that since the PRF is less than the azimuth Doppler bandwidth, the non-uniform real data are formed.

Table 2. Main parameters of real airborne data.

\begin{tabular}{cc}
\hline Parameter & Value \\
\hline Carrier Frequency & $1.3 \mathrm{GHz}$ \\
PRF & $149.92 \mathrm{~Hz}$ \\
Channel Number & 2 \\
Platform Velocity & $129.8 \mathrm{~m} / \mathrm{s}$ \\
Adjacent Channel Interval & $0.86 \mathrm{~m}$ \\
Slant Range & $1.58 \mathrm{~km}$ \\
Azimuth Band Width & $190.91 \mathrm{~Hz}$ \\
Range Band Width & $210 \mathrm{MHz}$ \\
Range Sampling Rate & $266 \mathrm{MHz}$ \\
Antenna Length & $1.36 \mathrm{~m}$ \\
\hline
\end{tabular}

In this experiment, three typical areas were selected for comparative analysis, as shown in the red box in Figure 9. Figure 9a presents the imaging result using the DBF algorithm to reconstruct the azimuth signal. It can be seen that the serious azimuth ambiguities are caused by non-uniform sampling and noise. In Figure 9b, the IDBF algorithm reduces azimuth ambiguity, but the imaging result is still not ideal. The imaging result using the proposed algorithm in this paper is shown in Figure 9c. The azimuth ambiguities denoted in red box can hardly be seen in Figure 9c, which means that the proposed algorithm can effectively suppress azimuth ambiguity. By comparing with the conventional algorithm and its improved algorithm, it becomes clear that the azimuth signal of MCSAR is well reconstructed based on the proposed algorithm. 


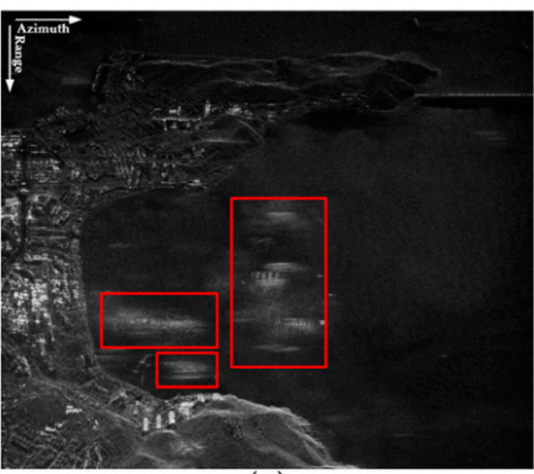

(a)

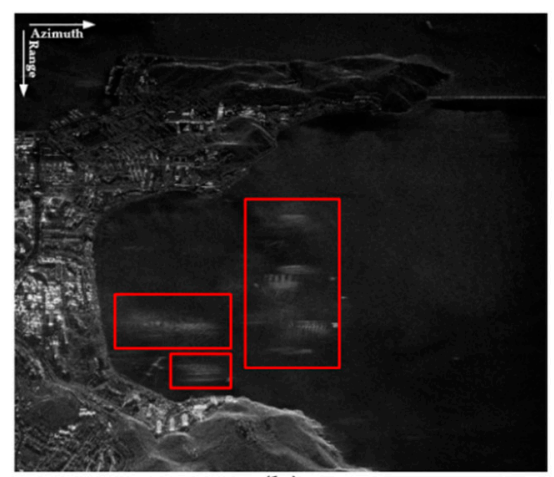

(b)

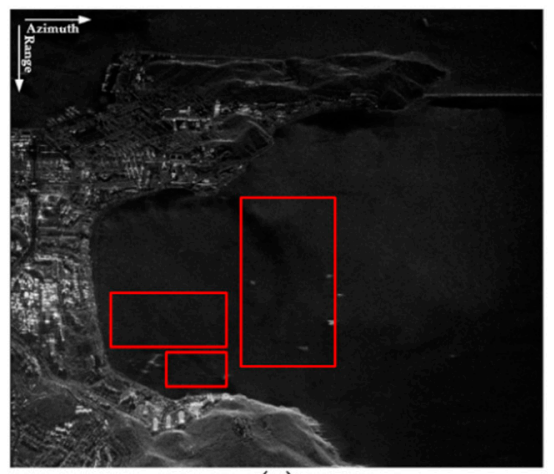

(c)

Figure 9. Real data processing: (a) DBF; (b) IDBF; (c) TSPT.

\section{Conclusions}

This paper proposes a novel reconstruction algorithm for azimuth MCSAR signals based on two-step projection technology. This algorithm performs a series of projections by introducing an intermediate subspace consisting of an integer shift of a tightly supported kernel. In the reconstruction process, the proposed algorithm can effectively eliminate the azimuth ambiguity caused by non-uniform sampling signals with noise. Simulation and real data processing results show that the proposed algorithm offers better SNR scaling and better AASR than the conventional algorithm and its improved algorithm. Therefore, the proposed algorithm can be used as a candidate algorithm for azimuth MCSAR signalreconstruction.

Author Contributions: Formal analysis, N.L. and H.Z.; methodology, N.L., Z.G. and H.Z.; validation, N.L., H.Z. and J.Z.; resources, N.L., J.Z., Z.G. and L.W.; writing-original draft preparation, H.Z.; writing-review and editing, N.L., L.W. and J.Z.; All authors have read and agreed to the published version of the manuscript.

Funding: This work was supported in part by the National Natural Science Foundation of China under grants 61871175 and 41901302, and in part by the Foundation of Key Laboratory of Radar Imaging and Microwave Photonics, Ministry of Education under grant RIMP2020003.

Acknowledgments: The authors would like to thank the anonymous reviewers for their valuable and detailed comments that are crucial in improving the quality of this paper.

Conflicts of Interest: The authors declare no conflict of interest.

\section{References}

1. Cumming, I.G.; Wong, F.H. Digital Processing of Synthetic Aperture Radar Data: Algorithms and Implementation; Artech House: Norwood, MA, USA, 2005.

2. Villano, M.; Krieger, G.; Moreira, A. Staggered SAR: High-Resolution Wide-Swath Imaging by Continuous PRI Variation. IEEE Trans. Geosci. Remote Sens. 2014, 52, 4462-4479. [CrossRef]

3. Kim, J.; Younis, M.; Prats-Iraola, P.; Gabele, M.; Krieger, G. First Spaceborne Demonstration of Digital Beamforming for Azimuth Ambiguity Suppression. IEEE Trans. Geosci. Remote Sens. 2013, 51, 579-590. [CrossRef]

4. Yang, J.; Qiu, X.; Zhong, L.; Shang, M.; Ding, C. A Simultaneous Imaging Scheme of Stationary Clutter and Moving Targets for Maritime Scenarios with the First Chinese Dual-Channel Spaceborne SAR Sensor. Remote Sens. 2019, 11, 2275. [CrossRef]

5. Xu, W.; Wei, Z.; Huang, P.; Tan, W.; Liu, B.; Gao, Z.; Dong, Y. Azimuth Multi-Channel Reconstruction for Moving Targets in Geosynchronous Spaceborne-Airborne Bistatic SAR. Remote Sens. 2020, 12, 1703. [CrossRef]

6. Curlander, J.; McDonough, R. Synthetic Aperture Radar: Systems and Signal Processing; Artech House: Hoboken, NJ, USA, 1991.

7. Gebert, N.; Almeida, F.; Krieger, G. Airborne demonstration of multichannel SAR imaging. IEEE Trans. Geosci. Remote Sens. Lett. 2011, 8, 963-967. [CrossRef]

8. Freeman, A.; Johnson, W.T.K.; Huneycutt, B.; Jordan, R.; Hensley, S.; Siqueira, P.; Curlander, J. The myth of the minimum SAR antenna area constraint. IEEE Trans. Geosci. Remote Sens. 2000, 38, 320-324. [CrossRef]

9. Krieger, G.; Gebert, N.; Moreira, A. Unambiguous SAR signal-reconstruction from nonuniform displaced phase center sampling. IEEE Geosci. Remote Sens. Lett. 2004, 1, 260-264. [CrossRef] 
10. Gebert, N.; Krieger, G.; Moreira, A. Digital Beam Forming for HRWS-SAR Imaging: System Design, Performance and Optimization Strategies. In Proceedings of the 2006 IEEE International Symposium on Geoscience and Remote Sensing, Denver, CO, USA, 31 July-4 August 2006; pp. 1836-1839. [CrossRef]

11. Gebert, N.; Krieger, G.; Younis, M.; Bordoni, F.; Moreira, A. Ultra Wide Swath Imaging with Multi-Channel ScanSAR. In Proceedings of the IEEE International Geoscience and Remote Sensing Symposium, Boston, MA, USA, 6-11 July 2008; pp. v21-v24. [CrossRef]

12. Zhang, Y.; Wang, W.; Deng, Y.; Wang, R. Signal-reconstruction Algorithm for Azimuth Multichannel SAR System Based on a Multiobjective Optimization Model. IEEE Trans. Geosci. Remote Sens. 2020, 58, 3881-3893. [CrossRef]

13. Krieger, G.; Gebert, N.; Moreira, A. Multidimensional Waveform Encoding: A New Digital Beamforming Technique for Synthetic Aperture Radar Remote Sensing. IEEE Trans. Geosci. Remote Sens. 2008, 46, 31-46. [CrossRef]

14. Gebert, N.; Krieger, G.; Moreira, A. Multi-channel Azimuth Processing in ScanSAR and TOPS Mode Operation. IEEE Trans. Geosci. Remote Sens. 2010, 48, 2994-3008. [CrossRef]

15. Zhao, S.; Wang, R.; Deng, Y.; Zhang, Z.; Li, N.; Guo, L.; Wang, W. Modifications on Multichannel Reconstruction Algorithm for SAR Processing Based on Periodic Nonuniform Sampling Theory and Nonuniform Fast Fourier Transform. IEEE J. Sel. Top. Appl. Earth Obs. Remote Sens. 2015, 8, 4998-5006. [CrossRef]

16. Younis, M.; Fischer, C.; Wiesbeck, W. Digital beamforming in SAR systems. IEEE Trans. Geosci. Remote Sens. 2003, 41, 1735-1739. [CrossRef]

17. Gebert, N.; Krieger, G. Azimuth Phase Center Adaptation on Transmit for High-Resolution Wide-Swath SAR Imaging. IEEE Trans. Geosci. Remote Sens. Lett. 2009, 6, 782-786. [CrossRef]

18. Currie, A.; Gebert, N.; Brown, M.A. Wide-swath SAR. IEE Proc. F Radar Signal Process. 1992, 139, 122-135. [CrossRef]

19. Li, Z.; Wang, H.; Su, T.; Bao, Z. Generation of wide-swath and high-resolution SAR images from multi-channel small spaceborne SAR systems. IEEE Geosci. Remote Sens. Lett. 2005, 2, 82-86. [CrossRef]

20. Li, Z.; Wang, H.; Bao, Z.; Liao, G. Performance improvement for constellation SAR using signal processing techniques. IEEE Trans. Aero. Elec. Sys. 2006, 42, 436-452. [CrossRef]

21. Liu, B.; He, Y. Improved DBF Algorithm for Multi-channel High-Resolution Wide-Swath SAR. IEEE Trans. Geosci. Remote Sens. 2016, 54, 1209-1225. [CrossRef]

22. Liu, N.; Wang, R.; Deng, Y.; Zhao, S.; Wang, X. Modified Multi-Channel Reconstruction Method of SAR with Highly Nonuniform Spatial Sampling. IEEE J. Sel. Top. Appl. Earth Obs. Remote Sens. 2017, 10, 617-627. [CrossRef]

23. Zuo, S.; Xing, M.; Xia, X.; Sun, G. Improved Signal-reconstruction Algorithm for Multi-channel SAR Based on the Doppler Spectrum Estimation. IEEE J. Sel. Top. Appl. Earth Obs. Remote Sens. 2017, 10, 1425-1442. [CrossRef]

24. Zhang, S.; Xing, M.; Xia, X.; Zhang, L.; Guo, R.; Liao, Y.; Bao, Z. Multi-channel HRWS SAR Imaging Based on Range-Variant Channel Calibration and Multi-Doppler-Direction Restriction Ambiguity Suppression. IEEE Trans. Geosci. Remote Sens. 2014, 52, 4306-4327. [CrossRef]

25. Gebert, N.; Krieger, G.; Moreira, A. Digital Beamforming on Receive: Techniques and Optimization Strategies for High-Resolution Wide-Swath SAR Imaging. IEEE Trans. Aero. Electron. Sys. 2009, 45, 564-592. [CrossRef]

26. Kiperwas, A.; Rosenfeld, D.; Eldar, Y.C. The SPURS Algorithm for Resampling an Irregularly Sampled Signal onto a Cartesian Grid. IEEE Trans. Med. Imaging 2017, 36, 628-640. [CrossRef] [PubMed]

27. Zhang, L.; Gao, Y.; Wang, K.; Liu, X. A Blind Reconstruction of Azimuth Signal for Multi-Channel HRWS SAR System. In Proceedings of the Proceedings of the 2017 IEEE Radar Conference (RadarConf), Seattle, WA, USA, 8-12 May 2017; pp. 1020-1023. [CrossRef]

28. Zhang, L.; Gao, Y.; Wang, K.; Liu, X. Azimuth Signal-Reconstruction for HRWS SAR from Recurrent Nonuniform Samples. In Proceedings of the 2016 CIE International Conference on Radar (RADAR), Guangzhou, China, 10-13 October 2016; pp. 1-4. [CrossRef]

29. Li, Z. Distributed Small Satellite SAR-InSAR-GMTI Signal Processing Algorithms. Ph.D. Thesis, Xidian University, Xi'an, China, 2006.

30. Zhang, S. High-Resolution and Wide-Swath Multi-Channel SAR and Moving Target Imaging Theory and Methods. Ph.D. Thesis, Xidian University, Xi'an, China, 2014.

31. Zhang, L. Azimuth Multi-Channel High-resolution and Wide-swath SAR Imaging Processing Technique. Ph.D. Thesis, Shanghai Jiao Tong University, Shanghai, China, 2018.

32. Davis, T.A.; Gao, Y.; Wang, K.; Liu, X. An unsymmetric-pattern multifrontal method. ACM Trans. Math. Softw. 2004, 30, 196-199. [CrossRef]

33. Davis, T.A. Direct Methods for Sparse Linear Systems; Artech House: Philadelphia, MA, USA, 2006.

34. Eldar, Y.C. Sampling Theory: Beyond Bandlimited Systems; Cambridge University Press: Cambridge, UK, 2015.

35. Zhao, P.; Deng, Y.; Wang, W.; Zhang, Y.; Wang, R. Ambiguity Suppression Based on Joint Optimization for Multi-channel Hybrid and $\pm \pi / 4$ Quad-Pol SAR Systems. Remote Sens. 2021, 13, 1907. [CrossRef] 